\title{
ARTICLE
}

Received 4 Mar 2015 | Accepted 23 Jul 2015 | Published 2 Sep 2015

DOI: $10.1038 /$ ncomms 9144

OPEN

\section{Realizing high figure of merit in heavy-band $p$-type half-Heusler thermoelectric materials}

Chenguang Fu', Shengqiang Bai ${ }^{2}$, Yintu Liu', Yunshan Tang ${ }^{2}$, Lidong Chen ${ }^{2}$, Xinbing Zhao ${ }^{1,3} \&$ Tiejun Zhu ${ }^{1,3}$

Solid-state thermoelectric technology offers a promising solution for converting waste heat to useful electrical power. Both high operating temperature and high figure of merit $z T$ are desirable for high-efficiency thermoelectric power generation. Here we report a high $z T$ of $\sim 1.5$ at 1,200 K for the p-type FeNbSb heavy-band half-Heusler alloys. High content of heavier $\mathrm{Hf}$ dopant simultaneously optimizes the electrical power factor and suppresses thermal conductivity. Both the enhanced point-defect and electron-phonon scatterings contribute to a significant reduction in the lattice thermal conductivity. An eight couple prototype thermoelectric module exhibits a high conversion efficiency of $6.2 \%$ and a high power density of $2.2 \mathrm{Wcm}^{-2}$ at a temperature difference of $655 \mathrm{~K}$. These findings highlight the optimization strategy for heavy-band thermoelectric materials and demonstrate a realistic prospect of high-temperature thermoelectric modules based on half-Heusler alloys with low cost, excellent mechanical robustness and stability.

\footnotetext{
${ }^{1}$ State Key Laboratory of Silicon Materials and School of Materials Science and Engineering, Zhejiang University, Hangzhou 310027, China. ${ }^{2}$ State Key Laboratory of High Performance Ceramics and Superfine Microstructure, Shanghai Institute of Ceramics, Chinese Academy of Sciences, Shanghai 200050, China. ${ }^{3}$ Key Laboratory of Advanced Materials and Applications for Batteries of Zhejiang Province, Zhejiang University, Hangzhou 310027, China. Correspondence and requests for materials should be addressed to L.D.C. (email: cld@mail.sic.ac.cn) or to T.J.Z. (email: zhutj@zju.edu.cn.).
} 
$\mathrm{T}$ he demand for sustainable energies has sparked significant research into different types of energy conversion technologies in the past decades. Thermoelectric materials, which can directly convert waste heat into usable electricity, have received more and more attention for promising application in energy harvesting ${ }^{1,2}$. The conversion efficiency $\eta$ of a thermoelectric device is limited by the Carnot efficiency $\eta_{c}$, and the figure of merit $z T$ of the thermoelectric materials, which is expressed as $z T=\alpha^{2} \sigma T /\left(\kappa_{\mathrm{e}}+\kappa_{\mathrm{L}}\right)$, where $\alpha, \sigma, T, \kappa_{\mathrm{e}}$ and $\kappa_{\mathrm{L}}$ are the Seebeck coefficient. respectively, the electrical conductivity, the absolute temperature and the electronic and lattice components of total thermal conductivity $\kappa$ (ref. 1 ). Thus, a high $\eta_{c}$ and a high $z T$ will result in enhanced conversion efficiency. The thermoelectric parameters $\alpha, \sigma$, and $\kappa_{\mathrm{e}}$ are intimately interrelated via carrier concentration and it has been a big challenge to decouple the thermal and electrical properties. Two main strategies, therefore, have been individually adopted to improve $z T$. One is to maximize the power factor $\alpha^{2} \sigma$ through optimal doping and band engineering $1,3,4$. The other targets to reduce the lattice thermal conductivity $\kappa_{\mathrm{L}}$ by nanostructuring or phonon engineering ${ }^{5,6}$.

Traditional good thermoelectric materials, such as $\mathrm{Bi}_{x} \mathrm{Sb}_{2-x} \mathrm{Te}_{3}$ alloys near room temperature, $\mathrm{PbTe}_{1-x} \mathrm{Se}_{x}$ alloys at moderate temperature and $\mathrm{Si}_{1-x} \mathrm{Ge}_{x}$ alloys at high temperature, have high carrier mobility $\mu$ and reduced $\kappa_{\mathrm{L}}$ (refs 7,8 ). A common character of these materials is that their band structures near the Fermi levels are dominated by the $s$ or $p$ electronic states, accounting for the low density of states effective mass $m^{*}$ and high $\mu$. These light-band thermoelectric semiconductors with small $m^{*}$ $\left(0.1 m_{\mathrm{e}}-1.0 m_{\mathrm{e}}\right)$ generally request relatively low-optimal carrier concentration $p_{\text {opt }}\left(10^{19}-10^{20} \mathrm{~cm}^{-3}\right)$, as shown in Fig. 1a, a low content of dopants is enough to optimize their power factors.

In recent years, some other semiconductors have also been identified as promising high-performance thermoelectric materials, such as tin selenides ${ }^{2}$, filled skutterudites ${ }^{9}$ and half-Heusler compounds ${ }^{10,11}$. Most of them contain transition metal elements, such as $\mathrm{Fe}, \mathrm{Co}, \mathrm{Ni}$ et al., and their localized $3 d$ states make the valence band maximum or conduction band minimum flat and heavy ${ }^{12,13}$. Typically, the $m^{*}$ of these heavyband materials are in the range of $2 m_{\mathrm{e}}-10 m_{\mathrm{e}}$ (Fig. 1a). Thus, higher carrier concentrations, which demands for higher contents of dopants, are necessary to optimize the power factors. For example, the $p_{\mathrm{opt}}$ of heavy-band $\mathrm{ZrNiSn}$ alloys is $\sim 4 \times 10^{20} \mathrm{~cm}^{-3}$, one order of magnitude higher than that of $\operatorname{PbTe}\left(\sim 3 \times 10^{19} \mathrm{~cm}^{-3}\right)$, while the $p_{\text {opt }}$ of filled $\mathrm{CoSb}_{3}$ and FeNbSb system with larger $m^{*}$ are above $10^{21} \mathrm{~cm}^{-3}$ (Fig. 1b). Note that even though these heavy-band thermoelectric materials have large $m^{*}$ and hence low $\mu$, their optimal power factors are 2-3 times higher than the state-of-the-art light-band $\mathrm{PbTe}$, which is an important reason making these heavy-band thermoelectrics promising for power generation. An immediate question arises that what is the effective optimization strategy for achieving high $z T$ heavy-band thermoelectric materials?

Alloying (substitution or doping) creates point-defect scattering for phonons due to mass fluctuation and strain field fluctuation between the host atoms and alloying atoms ${ }^{14}$, and results in reduced $\kappa_{\mathrm{L}}$. In thermoelectric materials, dopants not only supply carriers to optimize the power factor, but deduce point-defect scattering of phonons to suppress $\kappa_{\mathrm{L}}$. For light-band thermoelectric semiconductors, the $p_{\text {opt }}$ is relatively low and a slight content of dopants are enough to optimize the power factor ${ }^{15,16}$, and the dopants usually contribute less to the $\kappa_{\mathrm{L}}$ reduction. By contrast, in heavy-band semiconductors, higher contents of dopants are demanded for optimizing the carrier concentration to reach the same Femi level (Fig. 1c). For example, $\sim 20 \%$ Sn was doped to optimize the power factor of heavy-band
$\mathrm{ZrCoSb}$ compounds ${ }^{17}$. Such a high content of dopant will also definitely create strong point-defect phonon scattering to reduce $\kappa_{\mathrm{L}}$. Furthermore, stronger point-defect phonon scattering may occur if the doping atoms have larger mass and strain field fluctuations compared with the host atoms (Fig. 1c), which could be an effective strategy for simultaneously optimizing electrical power factor and reducing thermal conductivity in heavy-band thermoelectric materials.

A high Carnot limit, $\eta_{\mathrm{c}}=\left(T_{\mathrm{H}}-T_{\mathrm{C}}\right) / T_{\mathrm{H}}$, needs a large temperature difference between the temperature of hot side, $T_{\mathrm{H}}$, and temperature of cold side, $T_{\mathrm{C}}$, of the thermoelectric device. Therefore, high temperature thermoelectric materials with superior properties are highly desirable for power generation operating above $1,000 \mathrm{~K}$. Half-Heusler compounds have attracted more and more attention due to their good electrical and mechanical properties and thermal stability at high temperatures $^{11,17-26}$. The highest $z T$ s of $\sim 1.0$ have been reported for $n$-type ZrNiSn-based half-Heusler alloys ${ }^{18,20,21,24}$. But developing high-performance $p$-type Zr-based half-Heusler compounds is still a big challenge ${ }^{17,24}$. Recently, we found that $p$-type $\mathrm{Fe}(\mathrm{V}, \mathrm{Nb}) \mathrm{Sb}$-based heavy-band half-Heusler compounds show great potential as high-temperature thermoelectric materials and a high $z T$ of 1.1 has been reached at $1,100 \mathrm{~K}$ in $\mathrm{FeNb}_{1-x} \mathrm{Ti}_{x} \mathrm{Sb}$ with high Ti content up to $20 \%{ }^{19,27}$. Although the $\kappa_{\mathrm{L}}$ of Ti-doped FeNbSb is remarkably reduced due to the enhanced point-defect scattering, it is still $\sim 3$ times as high as the calculated minimum $\kappa_{\mathrm{L}}\left(\sim 1 \mathrm{~W} \mathrm{~m}^{-1} \mathrm{~K}^{-1}\right)^{19}$. To achieve higher $z T$ in $p$-type FeNbSb, it is imperative to further suppress its $\kappa_{\mathrm{L}}$. Based on the above consideration and Fig. 1c, selecting the high contents of doping atoms having larger mass and radius differences with the host atoms may lead to further $\kappa_{\mathrm{L}}$ reduction at optimal carrier concentration and hence enhanced $z T$.

Here we indeed demonstrate that the thermoelectric properties of $p$-type FeNbSb half-Heusler compound can be significantly enhanced through heavier $\mathrm{Hf}$ doping. A record-high $z T$ of up to 1.5 at $1,200 \mathrm{~K}$ has been obtained in the heavy-band $\mathrm{FeNb}_{1-x} \mathrm{Hf}_{x} \mathrm{Sb}$ alloys. High contents of $\mathrm{Hf}$ and $\mathrm{Zr}$ dopants result in enhanced point-defect scattering of phonons, and the $\mathrm{Hf}$ doping at $\mathrm{Nb}$ site leads to the stronger phonon scattering. Interestingly, the electron-phonon scattering is found to also strongly contribute to the reduced $\kappa_{\mathrm{L}}$ at high dopant contents. An eight $n-p$ couples prototype half-Heusler thermoelectric module, based on our high-performance $n$-type $\mathrm{ZrNiSn}$ (ref. 18) and $p$-type FeNbSb compounds, is successfully assembled for the first time in this work. A maximum conversion efficiency of $6.2 \%$ and a power density of $2.2 \mathrm{~W} \mathrm{~cm}^{-2}$ under a temperature difference of $655 \mathrm{~K}$ are achieved, exhibiting the great potential of low-cost p-type FeNbSb half-Heusler compounds for high temperature power generation.

\section{Results}

$z T$ enhancement and prototype half-Heusler module. Highquality $\mathrm{FeNb}_{1-x} \mathrm{Hf}_{x} \mathrm{Sb}$ and $\mathrm{FeNb}_{1-y} \mathrm{Zr}_{y} \mathrm{Sb} \quad(x, y=0-0.16)$ samples were fabricated by levitation melting and spark plasma sintering. X-ray diffraction (XRD) patterns show that the single phase products were obtained (Supplementary Fig. 1). Figure 2a shows the $z T$ values of these samples. A peak $z T$ of $\sim 1.5$ is reached at $1,200 \mathrm{~K}$ for $\mathrm{FeNb}_{0.88} \mathrm{Hf}_{0.12} \mathrm{Sb}$ and $\mathrm{FeNb}_{0.86} \mathrm{Hf}_{0.14} \mathrm{Sb}$, $\sim 40 \%$ higher than that of Ti-doped $\mathrm{FeNbSb}^{19}$, and the $z \mathrm{Ts}$ are remarkably higher than other well-known state-of-the-art p-type high-temperature thermoelectric materials over the whole temperature range. As known, the average $z T_{\text {avg }}$ is more important than the peak $z T$ for thermoelectric device application. The $z T_{\text {avg }}$ of $\mathrm{FeNb}_{0.88} \mathrm{Hf}_{0.12} \mathrm{Sb}$ sample is calculated to be $\sim 0.8$ and $\sim 1.0$ in the temperature range of $300-1,200$ and $500-1,200 \mathrm{~K}$, 
a

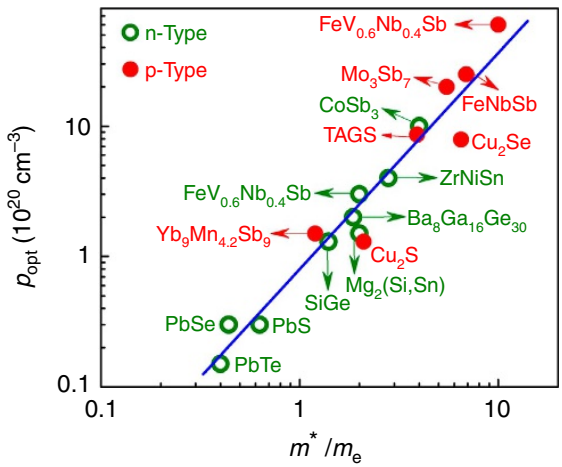

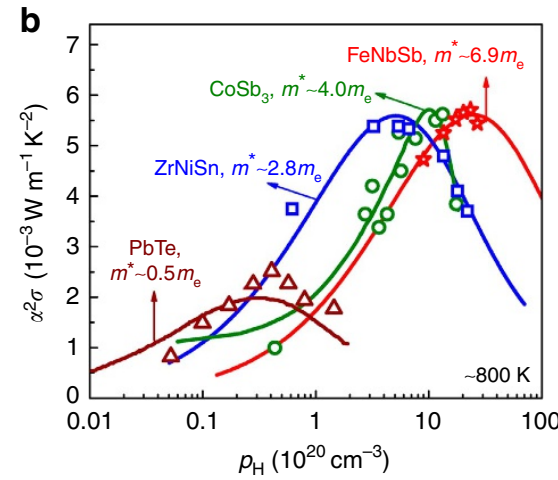

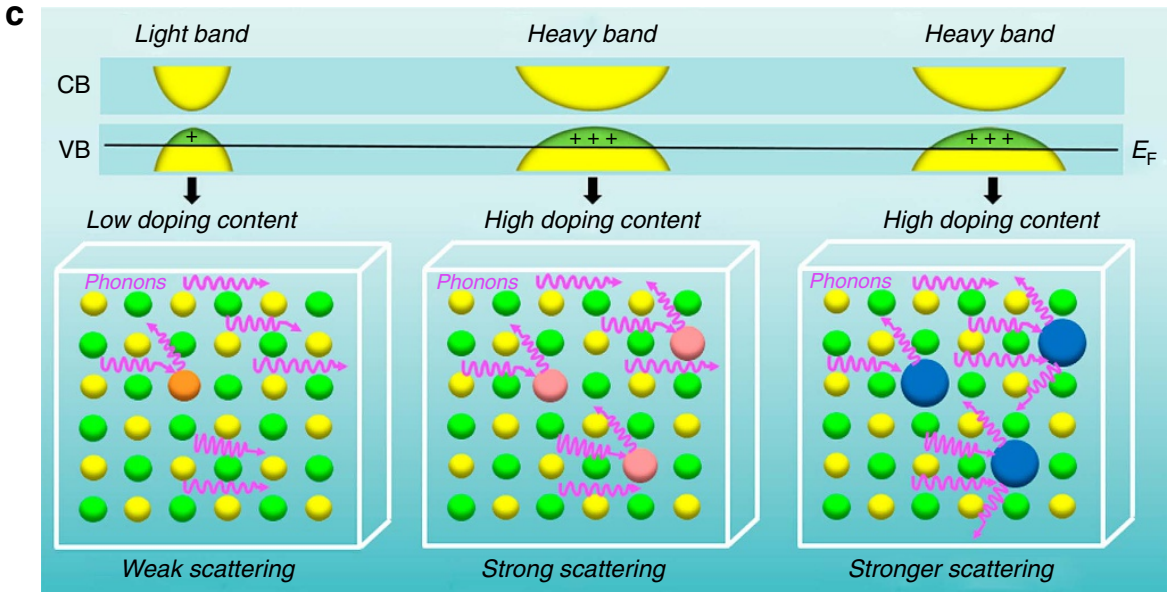

Figure 1 | Comparison of transport character of light-band and heavy-band thermoelectric materials. (a) The optimal carrier concentration $p_{\text {opt }}$ versus the density of state effective mass $m^{\star}$ for thermoelectric materials ${ }^{15,16,27,31-33,36,39-45}$. The solid line is a guide for eyes. (b) Carrier concentration dependence of power factor for the typical light-band $\mathrm{PbTe}^{15}$, and the heavy-band system: $n$-type $\mathrm{ZrNiSn}^{33}, n$-type filled $\mathrm{CoSb}_{3}{ }^{46}$ and $p$-type FeNbSb near $800 \mathrm{~K}$. (c) The schematic drawing shows the effect of band structure character on optimal doping content and hence phonon scattering.
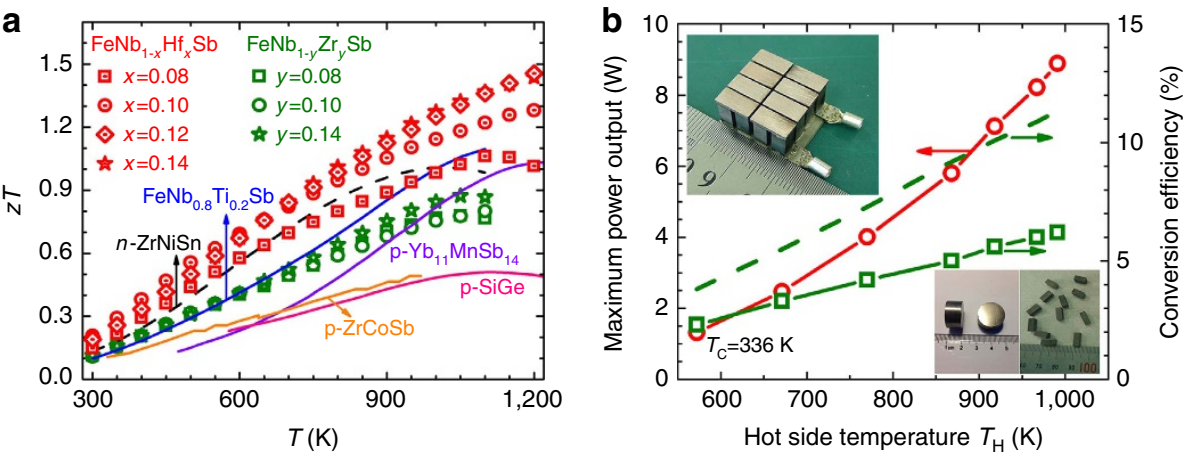

Figure 2 | Thermoelectric performance for p-type FeNbSb-based HH compounds and prototype module. (a) $z T$ comparison for $\mathrm{Hf}$ or $\mathrm{Zr}$ doped FeNbSb and other typical high temperature $p$-type thermoelectric materials ${ }^{17-19,40,47}$. (b) Maximum power output and conversion efficiency as a function of hot side temperature $T_{H}$ for the thermoelectric device made from our best $n$-type ZrNiSn-based alloys and $p$-type FeNbSb HH compounds. The dash line represents the theoretical conversion efficiency of the module with a maximum value of $11.3 \%$, assuming no electrical and thermal contact resistances.

respectively, even exceeding the industry benchmark set by conventional $p$-type SiGe alloys (peak $z T=0.6)^{17}$.

To corroborate the present results, the prototype hightemperature thermoelectric modules with eight $n-p$ half-Heusler couples were assembled (Fig. 2b) for the first time based on the best $n$-type $\mathrm{ZrNiSn}$-based alloys (thermoelectric properties are shown in Supplementary Fig. 2) and p-type FeNbSb compounds. The dimensions of the thermoelectric module made from the half-Heusler legs are $20 \mathrm{~mm}$ by $20 \mathrm{~mm}$ by $10 \mathrm{~mm}$ thick. Under conditions of hot/cold-side temperatures of $991 \mathrm{~K} / 336 \mathrm{~K}$, the halfHeusler module exhibited a maximum power output of $8.9 \mathrm{~W}$ and
$6.2 \%$ conversion efficiency, which is significantly higher than the conversion efficiency of $4.5 \%$ for the commercial half-Heusler modules based on $n$-type $\mathrm{ZrNiSn}$ and $p$-type $\mathrm{ZrCoSb}$-based half-Heusler alloys. Extrapolated values indicate that $8.1 \%$ is achievable when the hot-side temperature is up to $1,200 \mathrm{~K}$. The calculated total area power density for this half-Heusler module is about $2.2 \mathrm{~W} \mathrm{~cm}^{-2}$, which is significantly higher than other thermoelectric modules ${ }^{28-30}$ (Supplementary Table 1). The theoretical conversion efficiency is also calculated for comparison (dash line in Fig. 2b), which is higher than the experimental value. The discrepancy could be due to the 
matching between $n$-type and p-type legs, the insufficient contacting and the large radiation and convection losses and insufficient accuracy of measurement. Especially, the contact resistance contributes to about 3.2\% efficiency loss (Supplementary Discussion). More work is needed to improve the contacting electrical and thermal resistance and use thermal isolation between the half-Heusler legs.

Decoupling of electrical and thermal properties. Why do the p-type heavy-band $\mathrm{FeNb}_{1-x} \mathrm{Hf}_{x} \mathrm{Sb}$ alloys have so high $z T \mathrm{~s}$ ? The thermoelectric properties of $\mathrm{FeNb}_{1-x} \mathrm{Hf}_{x} \mathrm{Sb}$ and $\mathrm{FeNb}_{1-y} \mathrm{Zr}_{y} \mathrm{Sb}$ compounds are presented in Fig. 3, and analysed by using the single parabolic band (SPB) model ${ }^{31,32}$. The samples are heavily doped and the hole concentration is almost independent of temperature before intrinsic excitation (Supplementary Fig. 3). The electrical conductivity $\sigma$ of the $\mathrm{FeNb}_{1-x} \mathrm{Hf}_{x} \mathrm{Sb}$ and $\mathrm{FeNb}_{1-y} \mathrm{Zr}_{y} \mathrm{Sb}$ samples shows a metal-like behaviour and follows a temperature dependence of $T^{-1.5}$ (Fig. 3a), implying an acoustic phonon-scattering-dominated charge transport. The Seebeck coefficient $\alpha$ decreases with increasing carrier concentration (Fig. 3b). The calculated $\alpha$ by the SPB model agrees well with the experimental data before the intrinsic excitation. The $m^{*}$ was estimated to be $\sim 6.9 m_{\mathrm{e}}$ and almost unchanged at 300 and $800 \mathrm{~K}$, as shown in the Pisarenko plot of Fig. $3 c$, indicating that the valence band structure has weak dependence on temperature and the dopant type of Hf, $\mathrm{Zr}$ and $\mathrm{Ti}$.

The carrier concentration dependence of power factor for Hf- and Zr-doped FeNbSb samples at $800 \mathrm{~K}$ is shown in Fig. 3d, together with $\mathrm{Ti}$ doping data ${ }^{19}$. The optimal power factor ranges from 4.3 to $5.5 \times 10^{-3} \mathrm{~W} \mathrm{~m}^{-1} \mathrm{~K}^{-2}$ at $p_{\text {opt }}$ of $\sim 2 \times 10^{21} \mathrm{~cm}^{-3}$, which are relatively high values among established thermoelectric materials and comparable to the optimized $n$-type $\mathrm{ZrNiSn}$-based half-Heusler compounds ${ }^{33}$. Figure $3 \mathrm{~d}$ also indicates that the power factors of Hf-doped FeNbSb are higher than that of $\mathrm{Zr}$ - or Ti-doped samples. Further analysis shows that the Hf dopant is more efficient in supplying carriers than $\mathrm{Zr}$ and $\mathrm{Ti}$ (Supplementary Fig. 4). Thus at the carrier concentration of $\sim 2 \times 10^{21} \mathrm{~cm}^{-3}$ for $p$-type FeNbSb, the doping content of $\mathrm{Hf}, \mathrm{Zr}$ and $\mathrm{Ti}$ is about 12,14 and $16 \%$, respectively (Supplementary Fig. 4a). The corresponding room temperature carrier mobility for these samples are $18.4,15.0$ and $13.8 \mathrm{~cm}^{2} \mathrm{~V}^{-1} \mathrm{~s}^{-1}$, indicating that the less doping content for Hf-doped FeNbSb is beneficial for relatively higher carrier mobility due to the reduced alloy scattering of carriers. Therefore, at the same carrier concentration, the Hf-doped FeNbSb has higher power factors than $\mathrm{Zr}$ - and Ti-doped samples (Supplementary Fig. 4b). It is noteworthy that the different dopants also generate different effects on the thermal conductivity (Fig. 3d). The heavier Hf dopant leads to the $\sim 30 \%$ lower thermal conductivity compared with the $\mathrm{Zr}$ dopant, consistent with the discussion relevant to Fig. 1c.

Reduced lattice thermal conductivity and mechanisms. The

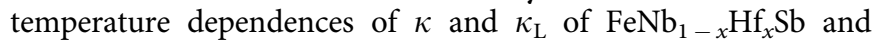
$\mathrm{FeNb}_{1-y} \mathrm{Zr}_{y} \mathrm{Sb}$ compounds are presented in Fig. 4. The $\kappa_{\mathrm{L}}$ was obtained by subtracting the electronic component $\kappa_{\mathrm{e}}$ from the total thermal conductivity $\kappa . \kappa_{\mathrm{e}}$ was calculated via WiedemannFranz relationship $\kappa_{\mathrm{e}}=L \sigma T$, where $L$ is the Lorenz number determined under the SPB approximation ${ }^{32}$. Figure 4 a shows the $\kappa$ of Hf- and $\mathrm{Zr}$-doped FeNbSb compounds are lower than that of FeNbSb. The decrease in $\kappa$ mainly results from the greatly suppressed $\kappa_{\mathrm{L}}$. As shown in Fig. $4 \mathrm{~b}$, with the same doping content, the $\kappa_{\mathrm{L}}$ of Hf-doped FeNbSb is lower than that of $\mathrm{Zr}$ - and Ti-doped samples, and the high-temperature $\kappa_{\mathrm{L}}$ of $\mathrm{FeNb}_{0.8} \mathrm{Ti}_{0.2} \mathrm{Sb}$ is only close to that of $\mathrm{FeNb}_{0.9} \mathrm{Hf}_{0.1} \mathrm{Sb}$, suggesting that $\mathrm{Hf}$ dopant leads to significantly reduced $\kappa_{\mathrm{L}}$ in FeNbSb even at a low content. The $\kappa_{\mathrm{L}}$ of $\mathrm{FeNb}_{1-{ }_{x}} \mathrm{Hf}_{x} \mathrm{Sb}$ decreases greatly with increasing $\mathrm{Hf}$ content. Especially, at 300 and $1,000 \mathrm{~K}$ the $\kappa_{\mathrm{L}}$ of $\mathrm{FeNb}_{0.86} \mathrm{Hf}_{0.14} \mathrm{Sb}$ has $\sim 80 \%$ and $\sim 70 \%$ reduction respectively, compared with
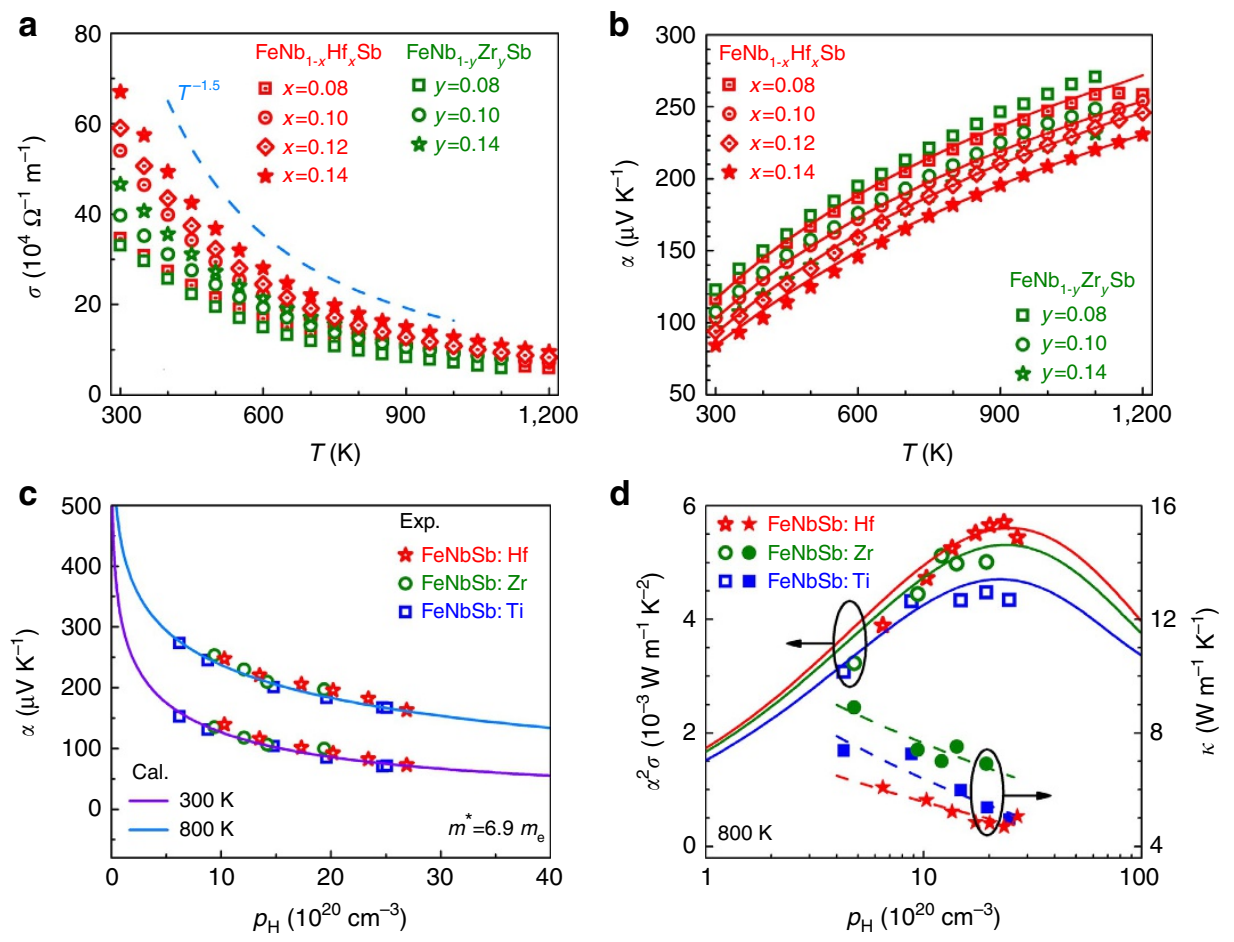

Figure 3 | Thermoelectric properties for FeNb $\mathbf{1}_{-\mathbf{x}} \mathbf{H f}_{\mathbf{x}} \mathbf{S b}$ and $\mathbf{F e N b _ { 1 } - \mathbf { z }} \mathbf{Z r}_{\mathbf{y}} \mathbf{S b}$ samples. (a) Electrical conductivity $\sigma$. (b) Seebeck coefficient $\alpha$. The $\alpha$ (c) and power factor $\alpha^{2} \sigma$ and thermal conductivity (d) of $\mathrm{Hf}$ - and Zr-doped FeNbSb as a function of carrier concentration, together with the data for Ti-doped $\mathrm{FeNbSb}^{19}$. The solid lines in $\mathbf{b}-\mathbf{d}$ were calculated by the SPB model. 

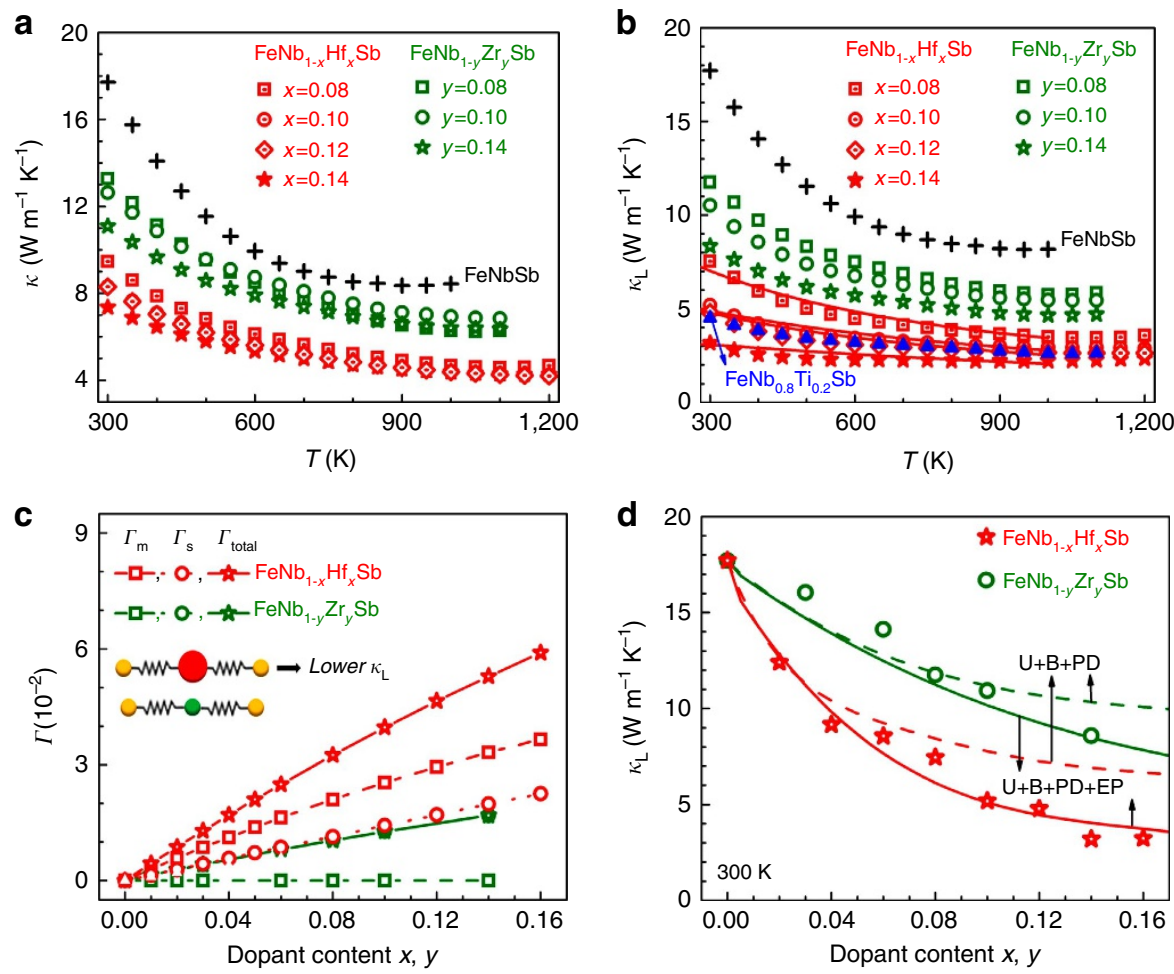

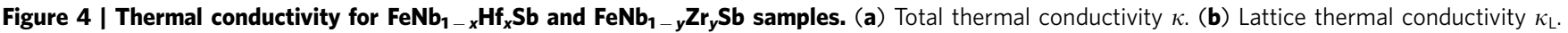
The solid curves in $\mathbf{b}$ are calculated using the Callaway model ${ }^{36,37}$. For comparison, $\kappa_{\mathrm{L}}$ of Ti-doped FeNbSb is also shown ${ }^{19}$. (c) The calculated disorder parameter $\Gamma$ for the samples, where $\Gamma_{\mathrm{m}}$ (square) and $\Gamma_{\mathrm{s}}$ (circle) are mass and strain field fluctuation disorder parameters, respectively. ${ }^{14,34}$ $\Gamma_{\text {total }}=\Gamma_{\mathrm{m}}+\Gamma_{\mathrm{s}}$. (d) Comparison of experimental and calculated $\kappa_{\mathrm{L}}$ for the samples at $300 \mathrm{~K}$. The dash and solid curves are calculated without and with electron-phonon scattering, respectively. U, B, PD and EP denote the phonon-phonon Umklapp process, boundary, point-defect and electron-phonon scattering, respectively.

that of FeNbSb, which is a key to the high $z T$ in this composition.

Why is Hf dopant more efficient in suppressing $\kappa_{\mathrm{L}}$ of FeNbSb despite of lower optimal content? As aforementioned, high content of dopants will create strong point-defect scattering of phonons, leading to the suppressed $\kappa_{\mathrm{L}}$. Hf doping at $\mathrm{Nb}$ sites will deduce more remarkable point-defect scattering than $\mathrm{Zr}$ and $\mathrm{Ti}$ because of the larger mass and radius differences between $\mathrm{Hf}$ and $\mathrm{Nb}$. For comparison, Fig. $4 \mathrm{c}$ presents the calculated disorder parameter $\Gamma$ (larger $\Gamma$ indicates stronger point-defect scattering of phonons ${ }^{14,34,35}$ ) for $\mathrm{Hf}$ and $\mathrm{Zr}$ at $\mathrm{Nb}$ sites, which obviously shows that the Hf creates stronger mass and strain field fluctuations, leading to lower $\kappa_{\mathrm{L}}$ in $\mathrm{FeNb}_{1-x} \mathrm{Hf}_{x} \mathrm{Sb}$.

The $\kappa_{\mathrm{L}}$ of the samples was further calculated by the Callaway model ${ }^{19,36,37}$. Phonon-phonon Umklapp process, grain boundary and point-defect scattering of phonons were firstly considered in the modelling. At low doping content, the calculated $\kappa_{\mathrm{L}}$ has a good agreement with the experimental results (Fig. 4d). However, at high doping contents, the calculated $\kappa_{\mathrm{L}}$ significantly deviates from the experimentally values, suggesting that some other scattering sources should also contribute to the reduced $\kappa_{\mathrm{L}}$ at high Hf or Zr contents. With increasing dopant content, the carrier concentration largely increases up to $10^{21} \mathrm{~cm}^{-3}$. The electronphonon interaction, an important part to scatter phonons in narrow semiconductors ${ }^{38}$, may exist in the $p$-type FeNbSb heavy-band system. With the electron-phonon scattering evolved, a good agreement between the experimental data and the calculated curves is reached (Fig. 4d). To corroborate this result, temperature dependence of $\kappa_{\mathrm{L}}$ was calculated for $\mathrm{FeNb}_{1-x} \mathrm{Hf}_{x} \mathrm{Sb}$ samples, and there is a good consistency with the experimental $\kappa_{\mathrm{L}}$ (Fig. $4 \mathrm{~b}$ ), indicating the enhanced electron- phonon scattering of phonons also contributes to the reduced $\kappa_{\mathrm{L}}$ for $\mathrm{FeNb}_{1-x} \mathrm{Hf}_{x} \mathrm{Sb}$ and $\mathrm{FeNb}_{1-y} \mathrm{Zr}_{y} \mathrm{Sb}$, especially at high doping contents. The similar phenomenon is also found in other thermoelectric materials ${ }^{36}$. Thus the simultaneously enhanced point-defect and electron-phonon scattering of phonons concurrently contribute to the reduced $\kappa_{\mathrm{L}}$ in the heavy-band $\mathrm{FeNb}_{1-x} \mathrm{Hf}_{x} \mathrm{Sb}$ system.

\section{Discussion}

In summary, by rationally selecting the heavier dopants at high contents, the interrelated thermoelectric parameters can be decoupled and the simultaneous optimization of electrical power factor and significant reduction in thermal conductivity can be achieved in heavy-band thermoelectric materials. Record-high $z T$ of 1.5 in $p$-type $\mathrm{FeNb}_{1-x} \mathrm{Hf}_{x} \mathrm{Sb}$ heavy-band half-Heusler compounds demonstrates the effective optimization strategy for achieving high thermoelectric performance. A prototype thermoelectric module made of $n$-type ZrNiSn-based alloys and $p$-type FeNbSb compounds exhibits a high conversion efficiency of $6.2 \%$ and a high power density of $2.2 \mathrm{~W} \mathrm{~cm}^{-2}$ at a temperature difference of $655 \mathrm{~K}$. These findings highlight the realistic prospect of high-temperature thermoelectric modules based on halfHeusler alloys with low cost, excellent mechanical properties and stability.

\section{Methods}

Synthesis. The ingots with nominal composition $\mathrm{FeNb}_{1-x} \mathrm{Hf}_{x} \mathrm{Sb}$ and $\mathrm{FeNb}_{1-} \mathrm{Zr}_{y} \mathrm{Sb}(x, y=0-0.16)$ were prepared by levitation melting of stoichiometric amount of $\mathrm{Fe}$ (piece, 99.97\%), Nb (foil, 99.8\%), Hf (piece, 99.99\%), Zr (foil, 99.99\%) and $\mathrm{Sb}$ (block, 99.999\%) under an argon atmosphere for several minutes. The ingots were remelted for four times to ensure homogeneity. The obtained 
ingots were mechanically milled (Mixer Mill MM200, Retsch) for $4 \mathrm{~h}$ under argon protection. The obtained powders were loaded into the graphite die and compacted by spark plasma sintering (SPS-1050, Sumitomo Coal Mining Co.) at 1,123 K for 10 min under $65 \mathrm{MPa}$ in vacuum. The as-sintered samples, of which the relative densities were found to be $\sim 95 \%$, were annealed at $1,073 \mathrm{~K}$ for 3 days.

Characterization. Phase structures of the samples were investigated by XRD on a RigakuD/MAX-2550PC diffractometer using $\mathrm{Cu} \mathrm{K}_{\alpha}$ radiation $\left(\lambda_{0}=1.5406 \AA\right)$. The XRD patterns of $\mathrm{FeNb}_{1-x} \mathrm{Hf}_{x} \mathrm{Sb}$ and $\mathrm{FeNb}_{1-y} \mathrm{Zr}_{y} \mathrm{Sb}$ show a single phase that can be indexed to the half-Heusler phase with a cubic MgAgAs-type crystal structure (space group, F43m) as shown in Supplementary Fig. 1. The lattice parameter of the samples increases with increasing dopant content as shown in Supplementary Fig. 5. The chemical compositions were checked by electron probe microanalysis (EPMA, JEOL and JXA-8100), which show that the actual compositions are close to the nominal ones (Supplementary Table 1). Scanning electron microscope and energy dispersive X-ray spectroscopy mapping were used to characterize the phase and compositional homogeneity (Supplementary Fig. 6). The average grain size of the sample was determined to be $\sim 0.8 \mu \mathrm{m}$ from the transmission electron microscope (FEI, Tecnai G2 F30 S-Twin) image (Supplementary Fig. 6).

Measurements. The Seebeck coefficient and electrical conductivity from 300 to $1,200 \mathrm{~K}$ were measured on a commercial Linseis LSR-3 system using a differential voltage/temperature technique and a d.c. four-probe method. The accuracy is $\pm 5 \%$ and $\pm 3 \%$, respectively. The thermal conductivity $\kappa$ was calculated by using $\kappa=D \rho C_{\mathrm{p}}$, where $\rho$ is the sample density estimated by the Archimedes method. The thermal diffusivity $D$ and specific heat $C_{\mathrm{p}}$ were measured by a laser flash method on Netzsch LFA457 instrument with a Pyroceram standard (Supplementary Fig. 7). The accuracy is $\pm 3 \%$ and $\pm 5 \%$, respectively. The low-temperature Hall coefficients from 20 to $300 \mathrm{~K}$ were measured using a Mini Cryogen Free Measurement System (Cryogenic Limited, UK). The carrier concentration $p_{\mathrm{H}}$ was calculated by $p_{\mathrm{H}}=1 /\left(e R_{\mathrm{H}}\right)$, where $e$ is the unit charge and $R_{\mathrm{H}}$ is the Hall coefficient. The estimated error of Hall coefficient is within $\pm 10 \%$. The carriers mobility $\mu_{\mathrm{H}}$ was calculated by $\mu_{\mathrm{H}}=\sigma R_{\mathrm{H}}$. The samples with highest $z T$ were repeatedly measured in Zhejiang University and Shanghai Institute of Ceramics, Chinese Academy of Science, and the results show good consistency (Supplementary Fig. 8). The high-temperature thermal stability of the sample was checked through the thermogravimetric analysis (Supplementary Fig. 9) and the accuracy is $5 \%$.

Thermoelectric module. For the eight $n-p$ couple prototype module assembly, the cylindrical half-Heusler pucks were diced into legs of square $4 \mathrm{~mm}$ by $4 \mathrm{~mm}$. Then the $n$-type and $p$-type half-Heusler legs were connected to metallic interconnects using high-temperature braze. The modules contain a total of 16 legs joined into 8 $n-p$ couples, all connected electrically in series and thermally in parallel. The power output, internal resistance and energy conversion efficiency of the half-Heusler prototype modules were evaluated in vacuum by using PEM-2 testing system (ULVAC-RIKO, Inc.). The electrodes coexist stably with $p / n$ half-Heusler alloys in the module's working temperature range from 300 to $1,000 \mathrm{~K}$. The accuracy of measurement for output power and conversion efficiency is about $10-15 \%$.

\section{References}

1. Snyder, G. J. \& Toberer, E. S. Complex thermoelectric materials. Nat. Mater. 7, 105-114 (2008)

2. Zhao, L. D. et al. Ultralow thermal conductivity and high thermoelectric figure of merit in SnSe crystals. Nature 508, 373-377 (2014).

3. Pei, Y. Z. et al. Convergence of electronic bands for high performance bulk thermoelectrics. Nature 473, 66-69 (2011).

4. Heremans, J. P. et al. Enhancement of thermoelectric efficiency in PbTe by distortion of the electronic density of states. Science 321, 554-557 (2008).

5. Biswas, K. et al. High-performance bulk thermoelectrics with all-scale hierarchical architectures. Nature 489, 414-418 (2012).

6. Toberer, E. S., Zevalkink, A. \& Snyder, G. J. Phonon engineering through crystal chemistry. J. Mater. Chem. 21, 15843-15852 (2011).

7. Ioffe, A. F. Semiconductor Thermoelements and Thermoelectric Cooling (Infosearch, 1957).

8. Goldsmid, H. J. Introduction to Thermoelectricity (Springer, 2010).

9. Sales, B. C., Mandrus, D. \& Williams, R. K. Filled skutterudite antimonides: a new class of thermoelectric materials. Science 272, 1325-1328 (1996).

10. Tritt, T. M. Holey and unholey semiconductors. Science 283, 804-805 (1999).

11. Xie, H. H. et al. Beneficial contribution of alloy disorder to electron and phonon transport in half-Heusler thermoelectric materials. Adv. Funct. Mater. 23, 5123-5130 (2013).

12. Yang, J. et al. Trends in electrical transport of $p$-type skutterudites $R \mathrm{Fe}_{4} \mathrm{Sb}_{12}$ $(R=\mathrm{Na}, \mathrm{K}, \mathrm{Ca}, \mathrm{Sr}, \mathrm{Ba}, \mathrm{La}, \mathrm{Ce}, \mathrm{Pr}, \mathrm{Yb})$ from first-principles calculations and Boltzmann transport theory. Phys. Rev. B 84, 235205 (2011).

13. Yang, J. et al. Evaluation of half-Heusler compounds as thermoelectric materials based on the calculation electrical transport properties. Adv. Funct. Mater. 18, 2880-2888 (2008).
14. Yang, J., Meisner, G. P. \& Chen, L. Strain field fluctuation effects on lattice thermal conductivity of $\mathrm{ZrNiSn}$-based thermoelectric compounds. Appl. Phys. Lett. 85, 1140-1142 (2004).

15. Pei, Y. Z. et al. Optimum carrier concentration in $\mathrm{n}$-type $\mathrm{PbTe}$ thermoelectrics. Adv. Energy Mater. 4, 1400486 (2014).

16. Wang, H., Pei, Y. Z., LaLonde, A. D. \& Snyder, G. J. Weak electron-phonon coupling contributing to high thermoelectric performance in n-type PbSe. Proc. Natl Acad. Sci. USA 109, 9705-9709 (2012).

17. Culp, S. R. et al. $\mathrm{Zr}, \mathrm{Hf}) \mathrm{Co}(\mathrm{Sb}, \mathrm{Sn})$ half-Heusler phases as high-temperature $\left(>700^{\circ} \mathrm{C}\right)$ p-type thermoelectric materials. Appl. Phys. Lett. 93, 022105 (2008).

18. Yu, C. et al. High performance half-Heusler thermoelectric materials $\mathrm{Hf}_{1-\mathrm{x}} \mathrm{Zr}_{\mathrm{x}} \mathrm{NiSn}_{1-\mathrm{y}} \mathrm{Sb}_{\mathrm{y}}$ prepared by levitation melting and spark plasma sintering. Acta Mater. 57, 2757-2764 (2009).

19. Fu, C. G., Zhu, T. J., Liu, Y. T., Xie, H. H. \& Zhao, X. B. Band engineering of high performance p-type FeNbSb based half-Heusler thermoelectric materials for figure of merit $z T>1$. Energy Environ. Sci. 8, 216-220 (2015).

20. Schwall, M. \& Balke, B. Phase separation as a key to a thermoelectric high efficiency. Phys. Chem. Chem. Phys. 15, 1868-1872 (2013).

21. Chen, S. et al. Effect of Hf concentration on thermoelectric properties of nanostructured $\mathrm{N}$-type half-Heusler materials $\mathrm{Hf}_{\mathrm{x}} \mathrm{Zr}_{1-\mathrm{x}} \mathrm{NiSn}_{0.99} \mathrm{Sb}_{0.01}$. $A d v$. Energy Mater. 3, 1210-1214 (2013).

22. Graf, T., Felser, C. \& Parkin, S. S. P. Simple rules for the understanding of Heusler compounds. Prog. Solid State Chem. 39, 1-50 (2011).

23. Chen, S. \& Ren, Z. Recent progress of half-Heusler for moderate temperature thermoelectric applications. Mater. Today 16, 387-395 (2013).

24. Xie, W. et al. Recent advances in nanostructured thermoelectric half-Heusler compounds. Nanomaterials 2, 379-412 (2012).

25. Schmitt, J., Gibbs, Z. M., Snyder, G. J. \& Felser, C. Resolving the true band gap of $\mathrm{ZrNiSn}$ half-Heusler thermoelectric materials. Mater. Horiz. 2, 68-75 (2015).

26. Joshi, G. et al. NbFeSb-based p-type half-Heuslers for power generation applications. Energy Environ. Sci. 7, 4070-4076 (2014).

27. Fu, C. G. et al. High band degeneracy contributing to high thermoelectric performance in p-type half-Heusler compounds. Adv. Energy Mater. 4, 1400600 (2014).

28. Bartholomé, K. et al. Thermoelectric modules based on half-Heusler materials produced in large quantities. J. Electron. Mater. 43, 1775-1781 (2014).

29. Mikami, M., Kobayashi, K. \& Tanaka, S. Power generation performance of thermoelectric module consisting of Sb-doped Heusler $\mathrm{Fe}_{2} \mathrm{VAl}$ sintered alloy. Mater. Trans. 52, 1546-1548 (2011).

30. Salvador, J. R. et al. Conversion efficiency of skutterudite-based thermoelectric modules. Phys. Chem. Chem. Phys. 16, 12510-12520 (2014).

31. Liu, X. H. et al. Low electron scattering potentials in high performance $\mathrm{Mg}_{2} \mathrm{Si}_{0.45} \mathrm{Sn}_{0.55}$ based thermoelectric solid solutions with band convergence. Adv. Energy Mater. 3, 1238-1244 (2013).

32. May, A. F., Toberer, E. S., Saramat, A. \& Snyder, G. J. Characterization and analysis of thermoelectric transport in $\mathrm{n}$-type $\mathrm{Ba}_{8} \mathrm{Ga}_{16-x} \mathrm{Ge}_{30+x}$. Phys. Rev. $B$ 80, 125205 (2009).

33. Xie, H. H. et al. The intrinsic disorder related alloy scattering in $\mathrm{ZrNiSn}$ half-Heusler thermoelectric materials. Sci. Rep. 4, 6888 (2014).

34. Wang, H., LaLonde, A. D., Pei, Y. Z. \& Snyder, G. J. The criteria for beneficial disorder in thermoelectric solid solutions. Adv. Funct. Mater. 23, 1586-1596 (2013).

35. Abeles, B. Lattice thermal conductivity of disordered semiconductor alloys at high temperatures. Phys. Rev. 131, 1906-1911 (1963).

36. Shi, X. Y., Pei, Y. Z., Snyder, G. J. \& Chen, L. Optimized thermoelectric properties of $\mathrm{Mo}_{3} \mathrm{Sb}_{7-\mathrm{x}} \mathrm{Te}_{\mathrm{x}}$ with significant phonon scattering by electrons. Energy Environ. Sci. 4, 4086-4095 (2011).

37. Callaway, J. Model for lattice thermal conductivity at low temperatures. Phys. Rev. 113, 1046-1051 (1959).

38. Delaire, O. et al. Phonon softening and metallization of a narrow-gap semiconductor by thermal disorder. Proc. Natl Acad. Sci. USA 108, 4725-4730 (2011).

39. Wang, H., Schechtel, E., Pei, Y. Z. \& Snyder, G. J. High thermoelectric efficiency of n-type PbS. Adv. Energy Mater. 3, 488-495 (2013).

40. Vining, C. B., Laskow, W., Hanson, J. O., Vanderbeck, R. R. \& Gorsuch, P. D. Thermoelectric properties of pressure-sintered $\mathrm{Si}_{0.8} \mathrm{Ge}_{0.2}$ thermoelectric alloys. J. Appl. Phys. 69, 4333-4340 (1991).

41. Fu, C. G. et al. Electron and phonon transport in Co-doped $\mathrm{FeV}_{0.6} \mathrm{Nb}_{0.4} \mathrm{Sb}$ half-Heusler thermoelectric materials. J. Appl. Phys. 114, 134905 (2013).

42. Pei, Y. Z. et al. Improving thermoelectric performance of caged compounds through light-element filling. Appl. Phys. Lett. 95, 042101 (2009).

43. Bux, S. K. et al. Glass-like lattice thermal conductivity and high thermoelectric efficiency in $\mathrm{Yb}_{9} \mathrm{Mn}_{4.2} \mathrm{Sb}_{9}$. J. Mater. Chem. A 2, 215-220 (2014).

44. He, Y. et al. High thermoelectric performance in non-toxic earth abundant copper sulfide. Adv. Mater. 26, 3974-3978 (2014). 
45. Zhu, T. J., Gao, H., Chen, Y. \& Zhao, X. B. Ioffe-Regel limit and lattice thermal conductivity reduction of high performance $\left(\mathrm{AgSbTe}_{2}\right)_{15}(\mathrm{GeTe})_{85}$ thermoelectric materials. J. Mater. Chem. A 2, 3251-3256 (2014).

46. Shi, X. et al. Multiple-filled skutterudites: High thermoelectric figure of merit through separately optimizing electrical and thermal transports. J. Am. Chem. Soc. 133, 7837-7846 (2011).

47. Brown, S. R., Kauzlarich, S. M., Gascoin, F. \& Snyder, G. J. $\mathrm{Yb}_{14} \mathrm{MnSb}_{11}$ : New high efficiency thermoelectric material for power generation. Chem. Mater. 18, 1873-1877 (2006).

\section{Acknowledgements}

We would like to thank Professor Xun Shi and Mr Dudi Ren from Shanghai Institute of Ceramics for the repeated measurement of thermoelectric properties and device simulation, respectively. This work was supported by the National Basic Research Program of China (2013CB632500), the Nature Science Foundation of China (51171171), the Program for New Century Excellent Talents in University (NCET-120495) and the Key Research Program of Chinese Academy of Sciences (KGZD-EW-T06).

\section{Author contributions}

C.F. and T.Z. designed the experiment. C.F. and Y.L. prepared the samples and carried out thermoelectric property measurements. C.F. and T.Z. analysed the experimental data and established the thermoelectric transport model. S.B., Y.T. and L.C. fabricated the prototype thermoelectric module and measured its conversion efficiency. C.F., S.B., L.C., X.Z. and T.Z. wrote the manuscript.

\section{Additional information}

Supplementary Information accompanies this paper at http://www.nature.com/ naturecommunications

Competing financial interests: The authors declare no competing financial interests.

Reprints and permission information is available online at http://npg.nature.com/ reprintsandpermissions.

How to cite this article: Fu, C. et al. Realizing high figure of merit in heavy-band $p$-type half-Heusler thermoelectric materials. Nat. Commun. 6:8144 doi: 10.1038/ncomms9144 (2015).

(c) (i) This work is licensed under a Creative Commons Attribution 4.0 International License. The images or other third party material in this article are included in the article's Creative Commons license, unless indicated otherwise in the credit line; if the material is not included under the Creative Commons license, users will need to obtain permission from the license holder to reproduce the material. To view a copy of this license, visit http://creativecommons.org/licenses/by/4.0/ 